\title{
'Learning-by-doing' - Formando en Competencias para el Emprendimiento. Estudio de Caso: Grado en Liderazgo Emprendedor e Innovación (LEINN)
}

\section{Vicent Gassóa ${ }^{\text {, Aitor Lizarza }}{ }^{\mathrm{b}}$ y Enrique Garcia ${ }^{\mathrm{c}}$}

aFlorida Universitària - Mondragon Team Academy, vgasso@florida-uni.es, bMondragon Unibertsitatea - Mondragon Team Academy, alizarza@mondragon.edu y ${ }^{c}$ Florida Universitària Mondragon Team Academy, egarcia@florida-uni.es.

\begin{abstract}
Entrepreneurship has become one of the main strategic axis for employment creation, competitiveness improvement and societal economic growth. This situation pushes the educational institutions to take on the challenge of developing the key competences for entrepreneurship in the training-learning processes. The objectives of this study are to evaluate the results of the case study methodology and its application - Bachelor Degree in Entrepreneurial Leadership and Innovation of Mondragon Team Academy - and to extract recommendations for the development of methodologies in relation to university training in Entrepreneurship. The results of this study demonstrate that current university training in entrepreneurship has significant results on the development of key competencies for entrepreneurship, level of employability and creation of new companies. Likewise, the use of learningby-doing methodologies facilitates the development of these competences. In this sense, a good praxis is the creation of Junior Companies, where students develop real entrepreneurship projects and manage their own organization; complementing therefore their theoretical skills with practical experience.
\end{abstract}

Keywords: competences, entrepreneurship, leadership, innovation, learningby-doing, team learning, self-management, junior company, international experience, university bachelor degree.

\section{Resumen}

El emprendimiento se ha convertido en uno de los ejes estratégicos principales para la creación de empleo, la mejora de la competitividad y el crecimiento económico de la sociedad. Esta situación empuja a las diferentes instituciones educativas a asumir el reto de desarrollar las competencias para el emprendimiento en el proceso de formación-aprendizaje. Los objetivos de este estudio son evaluar los resultados de la metodología y su aplicación del estudio de caso del Grado en Liderazgo Emprendedor e Innovación de Mondragon Team Academy y extraer recomendaciones para el desarrollo de metodologías en relación a la formación universitaria en emprendimiento. Los resultados de este estudio demuestran que la formación universitaria en emprendimiento actual tiene significantes resultados en el desarrollo de las 
competencias clave para el emprendimiento, nivel de empleabilidad y creación de nuevas empresas. Así mismo, el uso de metodologías "learning-by-doing" facilitan el desarrollo de estas competencias. En este sentido, una buena praxis es la creación de Junior Empresas donde los estudiantes desarrollan proyectos de emprendimiento reales y gestionan su propia organización; complementando así sus habilidades teóricas con la experiencia práctica.

Palabras clave: competencias, emprendimiento, liderazgo, innovación, learning-by-doing, aprendizaje en equipo, autogestión, junior empresa, experiencia internacional, grado universitario.

\section{Introducción}

El emprendimiento se ha convertido en uno de los ejes estratégicos principales para la creación de empleo, la mejora de la competitividad y el crecimiento económico de la sociedad (Comisión Europea, 2012). Esta situación empuja a las diferentes instituciones educativas a asumir el reto de desarrollar las competencias para el emprendimiento en el proceso de formación-aprendizaje. La propia Comisión Europea ha marcado objetivos específicos en torno a la formación en emprendimiento:

- Promocionar el desarrollo de cualidades en los estudiantes tales como la creatividad, la proactividad, y la toma de decisiones, riesgos y responsabilidades.

- Fomentar la capacidad de resolver problemas, incluyendo la capacidad de planificación, comunicación, cooperación y trabajo en red.

- Desarrollar la auto-confianza y la motivación por actuar, aprender a pensar de manera crítica e independiente, y adquirir la voluntad y la capacidad de aprender de forma autónoma.

- Aportar las competencias y conocimientos empresariales necesarios para poner en marcha una nueva empresa.

La educación universitaria tradicional de la rama de empresariales, si bien satisface las competencias necesarias para gestionar empresas existentes, es criticada por no ser adecuada para satisfacer las necesidades de un entorno empresarial altamente cambiante y para el desarrollo de nuevos negocios (Bechard y Toulouse, 1998; Mitra, 2002; Gibb 2002). Según Jones y English (2004), la educación universitaria en emprendimiento "requiere un estilo de educación orientado a la acción que fomente el aprendizaje experimental, la resolución de problemas, la creatividad, el aprendizaje centrado en proyectos y que soporte un método de auto-evaluación". Es importante que estos programas también contemplen fundamentos básicos de las disciplinas de gestión empresarial (Gibb 2002), como por ejemplo economía, marketing y finanzas. Así mismo, según Garavan (1994), la duración de estos programas debería de ser lo suficientemente amplia para permitir una buena adquisición de las 
competencias pertinentes, que exigen no solo la gestión de multi-tareas a nivel empresarial sino también la operativa y relación con clientes y personas de la empresa.

Hasta el momento, pocos estudios han analizado los resultados de las metodologías existentes en formación universitaria en emprendimiento (Brown 2000; Comisión Europea, 2012). La mayoría de estos estudios se centran bien en el contexto de los EEUU, en programas tradicionales de empresariales complementados en emprendimiento, $y / o$ en programas de corta duración ( $\leq 2$ años). Hay una necesidad de estudios adicionales analizando programas universitarios de grado en el contexto europeo en formación integral en emprendimiento, para mejorar el estado del arte de la metodología y contribuir mejor a la creación de empleo y el crecimiento económico a nivel nacional y europeo.

\section{Objetivos}

El principal objetivo de este estudio es analizar, a través de un estudio de caso, los resultados de la metodología utilizada en el contexto europeo en formación universitaria de grado integral en emprendimiento. Para este estudio se han analizado los resultados del Grado en Liderazgo Emprendedor e Innovación de Mondragon Team Academy.

Los objetivos específicos de este estudio son:

1. Evaluar los resultados de la metodología y su aplicación del estudio de caso en las siguientes dimensiones: (a) adquisición de competencias clave, (b) emprendimiento después de la graduación y (c) empleabilidad del individuo.

2. Extraer recomendaciones para el desarrollo de metodologías y toma de decisiones institucionales en relación a la formación universitaria en emprendimiento.

\section{Metodología}

\subsection{Recopilación y análisis de datos}

Los resultados presentados en este estudio se basan en los datos de: (1) encuestas de valoración del grado realizadas a las tres primeras generaciones de egresados del Grado en Liderazgo Emprendedor e Innovación de Mondragon Team Academy después de su graduación (2013-2015); (2) encuestas de los tutores de empresas en los Proyectos Fin de Grado de las tres primeras generaciones de egresados (2013-2015); (3) una sesión de grupo focal con 10 personas de las cuatro generaciones de egresados (2013-2016) para evaluar su perfil competencial y la experiencia en el grado (octubre, 2016); y (4) informe de empleabilidad de Mondragon Unibertsitatea (2016).

Los datos adquiridos se analizaron de acurdo a las siguientes dimensiones: Adquisición de competencias clave, Emprendimiento después de la graduación y Empleabilidad del individuo. La identificación de las competencias clave para el emprendimiento se basaron principalmente en Heinonen y Poikkijoki (2006), Chell y Rosemary (2009) y Comisión Europea (2007). 


\subsection{Descripción del Grado en Liderazgo Emprendedor e Innovación (LEINN)}

El Grado en Liderazgo Emprendedor e Innovación (LEINN) es un grado desarrollado e implantado desde el 2009/2010 por Mondragón Team Academy (MTA) - unidad de emprendimiento de la Facultad de Empresariales de Mondragon Unibertsitatea, País Vasco. Este grado es impartido en diferentes ciudades del mundo - Valencia, Bilbao, Oñati, Irún, Barcelona, Madrid, Querétaro y Shanghái. El Plan de estudios de este grado se estructura en un único ciclo compuesto por cuatro cursos que suman un total de 240 ECTS.

El objetivo general del grado es formar profesionales capacitados para liderar de forma eficiente la creación de empresas y equipos autogestionados destacando su capacidad como agentes de cambio en las empresas existentes y la generación y desarrollo de nuevas empresas, preparados para trabajar en un mundo globalizado y multilingüe, contribuyendo así al desarrollo de la sociedad del conocimiento cuya clave de competitividad sea la innovación.

\subsubsection{Características del grado}

A continuación, se detallan brevemente las características de formación propias de la titulación:

\section{Aprendizaje en equipo con empresas reales}

La metodología utilizada combina el ejercicio de las competencias personales individuales a través del trabajo en equipo. De esta forma se permite la identificación y consecución de resultados mayores a nivel individual al tiempo que se desarrolla la capacidad de aprendizaje y consecución de objetivos comunes en equipo. Es este sentido, se toma como referencia la teoría de "La quinta disciplina" de Senge (1998), la cual considera a las organizaciones como una integración de individuos que, al trabajar y aprender de forma conjunta, son más grandes que la suma de las partes individuales. Los alumnos forman empresas reales (Junior Empresas) de 10-15 personas, como herramientas de aprendizaje fundamental del grado cuyo fin es dotar a los alumnos de un entorno real donde experimentar durante los cuatro años, adquiriendo las competencias prácticas necesarias para la creación y gestión de proyectos empresariales. Parte de la adquisición de las competencias prácticas se realiza en estancias empresariales en el extranjero cada año, con una duración de 1 a 4 meses por curso en países como, por ejemplo, Finlandia, Estados Unidos, India y China.

Estos procesos de aprendizaje práctico son apoyados por formación teórica basada en la lectura de libros y asignaturas básicas, tales como matemáticas financieras, estadística aplicada, contabilidad, derecho de la empresa, economía de la empresa, ética empresarial, ingeniería de producto, informática (programación básica), inglés empresarial y chino conversacional. Los procesos de aprendizaje se llevan a cabo en

Las empresas necesitan profesionales con una visión global, dado que el mundo empresarial se fundamenta cada día más en la interacción entre países a nivel mundial y la actuación en mercados internacionales.

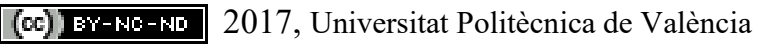




\section{Aprendizaje en espacios de emprendimiento e innovación empresarial}

Los alumnos desarrollan sus principales actividades de aprendizaje en espacios de emprendimiento e innovación empresarial (laboratorios de emprendimiento) con el objetivo de ofrecer un entorno que favorezca la experimentación, creatividad y desarrollo de nuevos conceptos y proyectos emprendedores. Físicamente, los laboratorios de emprendimiento son espacios con una estructura similar a los espacios de co-working (trabajo colaborativo). Funcionalmente, son "ecosistemas de emprendimiento e innovación”, donde a través de la colaboración los diferentes agentes pertenecientes al laboratorio (emprendedores, empresas, estudiantes, etc.), desarrollan colaboraciones, nuevas empresas e ideas de negocios contribuyendo al desarrollo económico y social del entorno.

\section{Alumnos protagonistas en el proceso de aprendizaje teórico-práctico}

La metodología de aprendizaje definida permite que el alumno asuma el papel primordial en el proceso de aprendizaje. De esta forma se evoluciona de un rol pasivo, controlado, teórico y sujeto a una evaluación y calificación puntual semestral a un rol activo, auto responsable, teórico-práctico y sujeto a un proceso de evaluación continuo y calificación semestral. Del mismo modo se asume un cambio en el rol del profesorado cuya función evoluciona de la de ponente magistral a la de un agente facilitador/coach del proceso de aprendizaje del alumno a nivel de equipo e individual. El coach tiene como principios, por ejemplo, fomentar el aprender haciendo, promover la confianza y el diálogo en el equipo, ayudar a convertir los conflictos en oportunidades y las diferencias en fortalezas y ayudar a alinear las energías para la consecución de una visión y objetivos compartidos.

La lectura de libros y la posterior elaboración de un informe/ensayo de cada uno es uno de los pilares del rol del alumno en el proceso de aprendizaje. De esta forma, el nuevo grado contempla que cada alumno deberá leer de media entre 40 y 60 libros a lo largo de la formación. Estos libros son elegidos por el alumno de una lista propuesta por materias y competencias siendo algunos de lectura obligatoria. Los alumnos desde sus empresas, como fruto de un trabajo individual y en equipo previo, llevarán a cabo proyectos con empresas, siendo ellos los protagonistas de todo el proceso y contando con el apoyo y supervisión de los tutores en el desarrollo de los proyectos. La existencia de un proceso de evaluación $360^{\circ}$ que incorpora la valoración de compañeros, coaches, profesores, clientes y proveedores asegura la evolución en el aprendizaje donde el alumno es máximo responsable y protagonista.

\section{Aprendizaje integral por procesos a través de un conocimiento implícito}

Se toma como modelo de referencia la teoría de gestión de conocimiento y aprendizaje organizacional (Nonaka y Tackeuchi, 1995) y su aplicación práctica en el modelo de enseñanza finlandés - Tiimiakatemia. Este modelo de aprendizaje integral por procesos es una evolución de la lógica histórica del aprendizaje por materias estancas y aisladas a un modelo de aprendizaje fundamentado en los procesos que permitan la interconexión entre materias, así como el desarrollo de competencias basado en el proceso del ciclo de vida de la creación de conocimiento "autorreflexión-experimentación-retroalimentación" (Nonaka y Tackeuchi, 1995). El programa del nuevo título dispone de mecanismos específicos 
(proyectos con empresas, evaluación $360 \%$, etc.) y herramientas (contrato de aprendizaje, sesiones de diálogo, etc.) que aseguren el paso del conocimiento explícito a un conocimiento implícito y propio por parte de los alumnos a través de la experiencia (Figura 1).

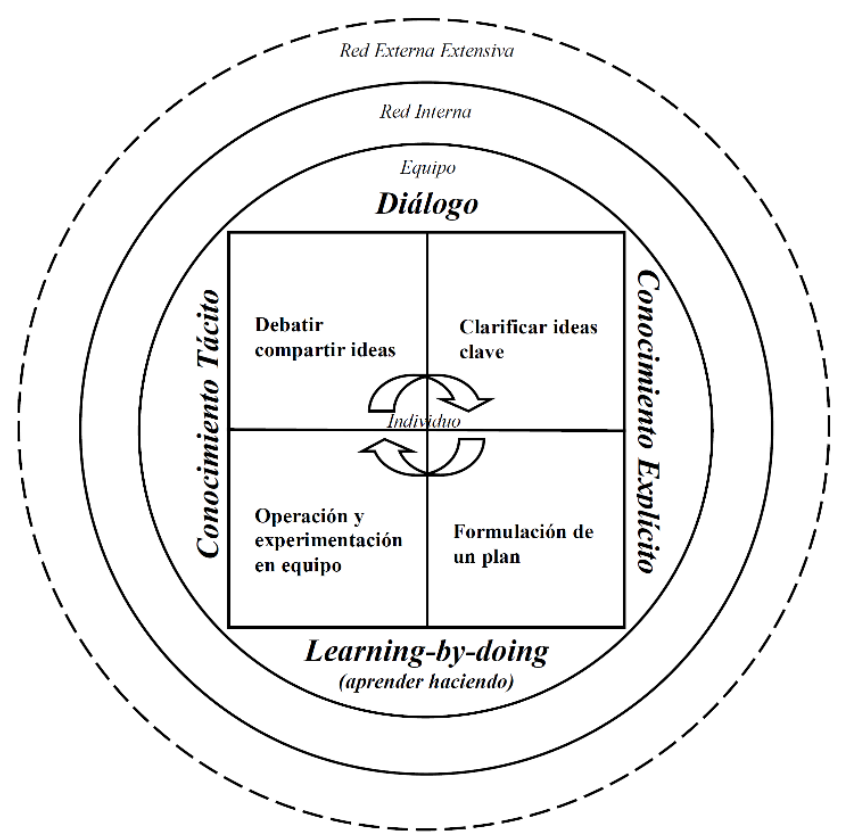

Fig. 1 Modelo de gestión de conocimiento en el Grado en Liderazgo Emprendedor e Innovación (Partanen, 2016 - adaptación de Nonaka y Takeuchi, 1995)

\subsubsection{Competencias del titulo}

Las competencias adquiridas por los graduados de esta titulación se agrupan en dos categorías:

- Competencias adquiridas a través del desarrollo de proyectos reales: (a) aprender en equipo, (b) liderar en equipo y (c) emprender en equipo (Tabla 1).

- Comprender e interpretar conocimientos acerca de: (a) la empresa y su entorno, (b) conocimientos instrumentales y (c) creación y gestión de empresas (Tabla 2).

Tabla 1. Competencias del grado Liderazgo Emprendedor e Innovación (LEINN) Categoría 1. Competencias adquiridas a través del desarrollo de proyectos reales

\section{Competencias adquiridas a través del desarrollo de proyectos reales}

\begin{tabular}{ll}
\hline Aprender en & - Tecnologías de la información y habilidades informáticas \\
equipo & - Técnicas de aprendizaje en equipo \\
& - Métodos personales de aprendizaje y actitudes (modelos mentales) \\
& - Creatividad \\
& - Visión internacional \\
& Habilidades comunicativas \\
\hline
\end{tabular}

(cc) EY-NC-ND 2017, Universitat Politècnica de València 


\begin{tabular}{ll}
\hline & - Auto-orientación \\
\hline Liderar en & - Habilidades de autogestión \\
equipo & - Gestión de proyectos \\
& - Capacidad de liderazgo de equipos \\
& - Habilidades de Coaching \\
& - Habilidades estratégicas \\
& - Toma de decisiones y determinación \\
\hline Emprender en & - Servicio al consumidor y técnicas de negociación y venta \\
equipo & - Comprensión de elementos financieros \\
& - Marketing \\
& - Innovación \\
& - Habilidades teóricas y de modelización de un emprendedor \\
& - Creación de redes o networking \\
&
\end{tabular}

Tabla 2. Competencias del grado Liderazgo Emprendedor e Innovación (LEINN) Categoría 2. Comprender e interpretar conocimientos

\begin{tabular}{|c|c|}
\hline \multicolumn{2}{|c|}{ Comprender e interpretar conocimientos } \\
\hline $\begin{array}{c}\text { La empresa y su } \\
\text { entorno }\end{array}$ & $\begin{array}{l}\text { - Principios de Microeconomía } \\
\text { - Características de la toma de decisiones y las teorías empleadas para } \\
\text { describirlas } \\
\text { - Naturaleza y comportamiento de la empresa } \\
\text { - Principios de Contabilidad } \\
\text { - Principios de Derecho Civil, Mercantil, Fiscal y Laboral } \\
\text { - Principios de ética }\end{array}$ \\
\hline $\begin{array}{l}\text { Conocimientos } \\
\text { Instrumentales }\end{array}$ & $\begin{array}{l}\text { - Principios de Matemática financiera y Estadística aplicada a la empresa } \\
\text { - Principales herramientas informáticas de aplicación en la empresa } \\
\text { - Principales herramientas de gestión de equipos, comunidades y trabajo en red } \\
\text { - Conocimientos de Inglés aplicado a la empresa } \\
\text { - Conocimientos de chino aplicado a la empresa }\end{array}$ \\
\hline $\begin{array}{l}\text { Creación y } \\
\text { gestión de } \\
\text { empresas }\end{array}$ & $\begin{array}{l}\text { - Proceso de creación de empresas, desde la conceptualización de la idea hasta } \\
\text { el desarrollo del plan estratégico de empresa y su puesta en marcha } \\
\text { - Gestión económica-financiera del desarrollo de nuevos negocios } \\
\text { - Gestión técnica de ingeniería de producto para el desarrollo de nuevos } \\
\text { productos y/o servicios } \\
\text { - Técnicas de desarrollo de la creatividad e identificación de oportunidades de } \\
\text { negocio y de innovación } \\
\text { - Técnicas de gestión de la innovación } \\
\text { - Técnicas de gestión de redes sociales y procesos de innovación abierta } \\
\text { - Principales técnicas de cálculo de costes y la consiguiente toma de decisiones } \\
\text { - Definición de la estrategia comercial, marketing y gestión de nuevos clientes } \\
\text { - Principales técnicas de selección de proyectos de inversión, estructura de } \\
\text { financiación, coste de capital, y análisis de riesgo-rentabilidad }\end{array}$ \\
\hline
\end{tabular}




\section{Resultados}

\subsection{Adquisición de competencias clave}

La evaluación que hacen los egresados en el informe de empleabilidad de Mondragon Unibertsitatea con respecto a la contribución de los estudios para el desarrollo de sus competencias profesionales es muy positiva. Asimismo, las empresas en las que han trabajado los estudiantes al realizar el Proyecto Fin de Grado, valoran muy positivamente sus competencias y consideran que su perfil es bueno para el desarrollo de actividades empresariales relacionados con el emprendimiento y la innovación. Además, los egresados consiguen numerosos contratos y colaboraciones posteriores en aquellas empresas donde realizan el Proyecto Fin de Grado.

Los egresados destacan y valoran como 'fuerte' la adquisición de competencias de visión internacional, iniciativa, pensamiento crítico, resolución de problemas, aprender a aprender, la autonomía responsable, y 'aceptable a reforzar' la adquisición de competencias tales como empatía, diversidad, humildad y atracción de inversión. Los alumnos reconocen la metodología de aprendizaje como diferenciadora y que les permite obtener las principales competencias clave de forma satisfactoria.

Otros estudios han demostrado que la formación universitaria en emprendimiento tiene un impacto positivo en las competencias clave (Comisión Europea, 2012). En estos estudios, los egresados formados en emprendimiento, en comparación con los egresados sin esta formación, se identifican más en que la educación superior contribuyó al desarrollo de actitudes relacionadas con la iniciativa, propensión al riesgo y necesidad de alcanzar logros. Sin embargo, los egresados formados en emprendimiento puntúan más bajo en actitudes relacionadas con la habilidad de trabajar de manera estructurada y en la capacidad de perseverar frente a retrocesos y obstáculos. En estos estudios no se han encontrado diferencias significantes con respecto al nivel de autoeficacia. Los egresados en emprendimiento puntúan más alto en competencias relacionadas con creatividad, capacidad analítica, capacidad de adaptación, capacidad de motivar a otros y con habilidades de networking y trabajo en red. Además, se identifican más en que la educación superior les ha dado las habilidades y el conocimiento que les permite manejar un negocio y ha contribuido a su comprensión del papel de los empresarios en la sociedad.

En los estudios revisados (Comisión Europea, 2012), los programas de formación utilizando metodologías learning-by-doing, como la creación de Junior Empresas, puntúan mejor en muchos aspectos relacionados con las competencias clave, en comparación con los programas de formación en emprendimiento sin este tipo de metodologías.

(cc) EY-NC-ND 2017, Universitat Politècnica de València 


\subsection{Empleabilidad del individuo}

La tasa de empleo de los egresados durante los cursos 2012/13, 2013/14 y 2014/15 muestra unos datos muy positivos sobre la inserción laboral. Específicamente, de los egresados encuestados en cada curso, un $88.9 \%, 88.5 \%$ y 91,7\% están trabajando en la actualidad, respectivamente. Estos datos son especialmente significantes considerando que el desempleo en menores de 25 años con formación superior en España es aproximadamente del 37\% (INE, 2015). La salida laboral del egresado LEINN puede bien enfocarse en el desarrollo de proyectos emprendedores propios o en la incorporación a departamentos de empresas ya consolidadas. La mayoría de los egresados de LEINN están trabajando en aquellas actividades relacionadas con el grado; es decir, en temas de liderazgo, emprendimiento/intraemprendimiento e innovación, incluyendo áreas como marketing, ventas, educación, negocios digitales y diseño.

Otros estudios también han demostrado que la formación universitaria en emprendimiento tiene un efecto positivo en la empleabilidad de los individuos, especialmente en términos de experiencia laboral, creatividad en el puesto de trabajo e ingresos anuales (Comisión Europea, 2012). Según estos estudios, parece ser más fácil para los individuos con formación en emprendimiento encontrar empleo inmediatamente después de su graduación y es menor la posibilidad de estar desempleados en los primeros años después de la graduación. En la muestra de estos otros estudios, entre el 78 y el $66 \%$ de los egresados en formación en emprendimiento indican que han comenzado su primer período de empleo inmediatamente después de su graduación, frente al 59\% de los egresados sin este tipo de formación.

\subsection{Emprendimiento después de la graduación}

Después de cuatro generaciones egresadas con un total de 150 graduados, se han creado un total de 14 empresas cofundadas en equipo por egresados de LEINN (no se incluyen aquellas empresas en las que los egresados se incorporan a equipos emprendedores y existentes). Más de 75 personas están directamente implicadas en el desarrollo de estos proyectos emprendedores por lo que desde la perspectiva de generación de empleo directo es significativo. El ritmo de creación es de 3,5 empresas por año y de una empresa por cada 10 graduados. Los equipos de emprendedores en las empresas creadas están formados por aproximadamente por 5 personas por empresa. La mayoría de las empresas (64\%) tienen una fuerte vocación de crecimiento con modelos de negocio que son escalables, además de ser interesantes para la inversión; con potencial de impacto en la economía y sociedad. Por otro lado, algunos proyectos están más enfocados en el autoempleo y micropymes (36\%). Predominan las empresas de servicios avanzados y las digitales, y varias de ellas incorporan tecnología en sus proyectos.

Otros estudios han demostrado que la formación universitaria en emprendimiento estimula el emprendimiento y el auto-empleo (Comisión Europea, 2012). En la muestra de estos estudios, el 16\% de los egresados con formación universitaria en emprendimiento son autoempleados, con respecto al $10 \%$ de los egresados sin esta formación. Además, los primeros comienzan en su primera empresa antes de la graduación ( 0,7 años de promedio) y los 
segundos comienzan después de la graduación (2,8 años de promedio). Los egresados con formación en emprendimiento también suelen tener ingresos netos anuales mayores que los egresados en otros programas. Así mismo, los egresados en programas en emprendimiento valoraron más su empresa como innovadora, en cuanto a introducir nuevos productos y servicios, o introducir nuevos procesos y formas de organización y prácticas empresariales.

Estos estudios también han demostrado que la formación universitaria en emprendimiento también estimula las intenciones de los estudiantes para convertirse en empresarios (Comisión Europea, 2012). Las principales razones para auto-emplearse expuestas por los estudiantes con formación en emprendimiento son la realización de una oportunidad de negocio y el deseo de independencia personal. Por otro lado, las razones para auto-emplearse expuestas por los estudiantes de otros programas son la falta de oportunidades de empleo atractivas y evitar la incertidumbre relacionada con ser empleado; es decir, más debido a las desventajas de ser empleado que a las ventajas de ser auto-empleado, como ocurre con los estudiantes con formación en emprendimiento. Los alumnos que prefieren ser empleados lo atribuyen a razones de seguridad y estabilidad, como disponer de ingresos regulares y fijos, horarios fijos, protección por la seguridad social y posibles problemas con las autoridades públicas.

\section{Conclusiones}

El uso de metodologías learning-by-doing en programas de formación universitaria en emprendimiento, como en el estudio de caso del grado LEINN, facilitan en los alumnos el desarrollo de las competencias clave para el emprendimiento. Asimismo, el perfil competencial de los formados en este tipo de metodologías presenta un gran potencial en términos de empleabilidad y creación efectiva de nuevas empresas.

La capacitación práctica debe de considerarse como ingrediente clave en estos programas de formación centrados en la adquisición de competencias. En este sentido, una buena práctica es la creación de Junior Empresas donde los estudiantes desarrollan proyectos de emprendimiento reales, se enfrentan a la relación con clientes y lideran y gestionan su propia organización en equipo; complementando así sus habilidades teóricas con la experiencia práctica.

El apoyo al desarrollo de la formación en emprendimiento en instituciones de educación superior debe de considerarse como clave para favorecer la creación de nuevos puestos de trabajo, así como mejorar su calidad e impacto positivo en la sociedad.

\section{Referencias}

BECHARD, J.P. y TOULOUSE, J.M. (1998). "Validation of a didactic model for the analysis of training objectives in entrepreneurship" en Journal of Business Venturing, vol. 13, issue 4, p. 317-332.

BROWN, C. (2000). Entrepreneurial Education Teaching Guide. CELCEE Digest 00-7. Adjunct ERIC Clearinghouse on Entrepreneurship Education, Los Angeles, CA.

(c) EY-NC-ND 2017, Universitat Politècnica de València 
CHELL, E. y ROSEMARY, A. (2009). The identification and measurement of innovative characteristics of young people: development of the Youth Innovation Skills Measurement Tool. London: NESTA. http://socialwelfare.bl.uk/subject-areas/services-client-groups/children-youngpeople/nesta/1516842009_identification_measurement_innovative_characteristics_young.pdf [Consulta: 20 de mayo de 2017].

COMISIÓN EUROPEA (2007). Key competences for lifelong learning, European Reference Framework. Luxemburgo. <https://erasmusplus.org.uk/file/272/download > [Consulta: 20 de mayo de 2017].

COMISIÓN EUROPEA (2012). Effects and impact of entrepreneurship programmes in higher education. Bruselas. [Consulta: 20 de mayo de 2017].

EUROSTAT (2015). Encuesta Europea de Fuerza del Trabajo (LFS) [Consulta: 20 de mayo de 2017].

GARAVAN, T.N. (1994). "Entrepreneurship Education and Training Programmes: A Review and Evaluation" en Journal of European Industrial Training, vol. 18, issue 11, p. 13-21.

GIBB, A.A. (2002) “Creating Conducive Environments for Learning and Entrepreneurship; living with, dealing with, creating and enjoying uncertainty and complexity" en Industry and Higher Education, vol. 16 , issue 3 .

HEINONEN, J. y POIKKIJOKI, S.A. (2006). An entrepreneurial-directed approach to entrepreneurship education: mission impossible? en Journal of Management Development, vol. 25, issue 1.

JONES, C. y ENGLISH, J. (2004) "A contemporary approach to entrepreneurship education", en Education + Training, vol. 46, issue 8/9, p. 416-423.

MITRA, J. (2002). "Consider Velasquez: reflections on the development of entrepreneurship programmes" en Industry and Higher Education Journal, vol. 13, issue 4, p. 317-332.

NONAKA, I., TAKEUCHI, H. (1995). La Organización Creadora de Conocimiento. Oxpford: Oxford University Press.

PARTANEN, J. (2016). The Team Coach's Best Tools. Jyväskylä: Partus.

SENGE, P. (1998). La Quinta Disciplina: Como Impulsar el Aprendizaje en la Organización Inteligente. Hospitalet de Llobregat: Ediciones Granica. 